\title{
Von der Routine zum Prozess
}

\begin{abstract}
Wir haben das Jahr 2022. Sie schlagen Ihre Morgenzeitung auf und folgende Überschrift erregt lhre Aufmerksamkeit: Die Entscheidung ist gefallen - die Anzahl der jährlich zugelassenen Innovationen wird aufgrund von Nachhaltigkeitsaspekten begrenzt! Was denken Sie? Utopie! Unmöglich! Oder vielleicht: Endlich? Natürlich wissen wir nicht, was 2022 in der Zeitung steht. Wir wissen nur, dass Innovationen nachhaltiger werden müssen. Wie könnte es nun Unternehmen im raven Markłwind gelingen, eine Strategie- und Innovationsbrücke zur Nachhaltigkeit zu bauen?
\end{abstract}

$\mathrm{V}$

Von Marlen Arnold und Andreas Aulinger

iele Ansätze des strategischen Managements und des Innovationsmanagements beschreiben den Ablauf von Strategie- oder Innovationsprozessen als scheinbar standardisierbare Abläufe. Dies geschieht entweder als Versuch einer Abbildung der Realität oder als Vorschlag dafür, wie Strategie- oder Innovationsprozesse in Unternehmen sinnvoll ablaufen sollten. Ein im Rahmen des Projektes Sustainable Markets Emerge (SUMMER) der Universität Oldenburg entwickeltes Modell nimmt hier eine neue Position ein. Es unterstellt keinen festen, gegebenenfalls einmaligen Durchlauf durch bestimmte Schritte, sondern orientiert sich an verschiedenen, auch oft wiederholenden Prozessen, die parallel, sequenziell oder zirkulär ablaufen können. Damit wird eine variantenreichere Sicht auf Strategie- oder Innovationsprozesse sowie die Reflektion darüber ermöglicht, welche Arten von Prozessen im eigenen Unternehmen existieren, welche dominieren und welche vielleicht auch gar nicht vorkommen. Damit kann das Modell eine gerichtete Kommunikation anregen und bereits auf diesem Wege Wirkung entfalten. Konkrete Gestaltungskraft erhält es zudem, wenn einzelne Teilprozesse oder bestimmte Kombinationen vertieft werden, um sie im Hinblick auf die Entwicklung nachhaltiger Zukunftsmärkte effizient einzusetzen.

\section{- Fünfstufiges Prozessmodell}

Im Folgenden geben wir einen Überblick über die grundsätzliche Bedeutung der fünf Prozesse und ihren Bezug zueinander. Alltagsprozesse (Grafik Punkt 1) existieren als einzige der fünf Teilprozesse in jedem Unternehmen. Sie sind gekennzeichnet durch vielfältige (Inter-)Aktionen, durch organisationale
Routinen, Reproduktionen und Reflexionen von Regeln, Prinzipien etc.. In diesen Prozessen finden viele kleine Lern- und Veränderungsschritte statt. Es werden täglich Entscheidungen getroffen und umgesetzt. Aus den Erfahrungen mit dieser Umsetzung kann für die nächsten Entscheidungen gelernt werden: ein bestimmter Lieferant wird mehr und mehr bevorzugt, ein anderer wird nicht mehr beauftragt; ein Produkt wird laufend verbessert; ein Organisationsablauf wird sukzessive optimiert. Manche Unternehmen realisieren im Rahmen dieser Alltagsprozesse - bewusst oder unbewusst - ihr gesamtes strategisches Management sowie Innovationsmanagement. Besonders in kleinen Unternehmen fehlen oft die Zeitreserven und auch das Methodenwissen, um in die anderen Prozesse einzusteigen. Veränderungsschritte, die innerhalb der Alltagsprozesse realisiert werden, bleiben dann zumeist sehr gering.

Erst wenn das Tagesgeschäft beiseite gelassen wird, Veränderungen gesucht werden, explizit nach der eigenen Situation in den aktuellen Märkten oder neuen Marktideen gefragt wird, entsteht das, was gemeinhin als eigentlicher Strategie- oder Innovationsprozess bezeichnet wird. Wir unterscheiden hier zwischen einem Prozess der ökonomischen Bewertung aktueller Märkte oder neuer Marktideen, kurz Strategie- und Innovationsbewertung (Grafik Punkt 2). Daneben steht der Prozess der konkreten Auswahl, Entwicklung und Umsetzung neuer Marktideen, kurz Strategie- und Innovationsrealisierung (Grafik Punkt 3). Die SUMMER-Projektpartner Velotaxi, ein innerstädtischer Mobilitätsdienstleister, und KonnexX, ein kleines Netzwerk von Schreinerein, haben als kleines Unternehmen und Unternehmensnetzwerk langjährig den Prozess eins durchlaufen. Mit externer Unterstïtzung konnte der zweite Prozess in Form von Strategietreffen dauerhaft im Unternehmen beziehungsweise Unternehmensnetzwerk verankert werden. Die monatlichen Strategietreffen bieten in der Hektik und dem „Chaos” des operativen Geschäftes die Chance zu Reflexion, zum Austausch wichtiger strategischer Informationen, zur Überprüfung und Abstimmung individueller Einschätzungen und zur Entwicklung gemeinsamer Sichtweisen, Einschätzungen und Strategien. Damit die Strategie- und Innovationsrealisierung auch erfolgreich ist, dienen dem SUMMER-Projektpartner BSH Bosch und Siemens Hausgeräte beispielhaft Business-InnovationsWorkshops, Technologie-Roadmapping sowie interaktive Konsumenten- und Anwenderforschung für die erfolgreiche Realisierung von Neuerungen im Bereich Kühlen. Mittels dieser Methoden und Instrumente werden zielgerecht innovative Projektideen ausgewählt, entwickelt und umgesetzt.

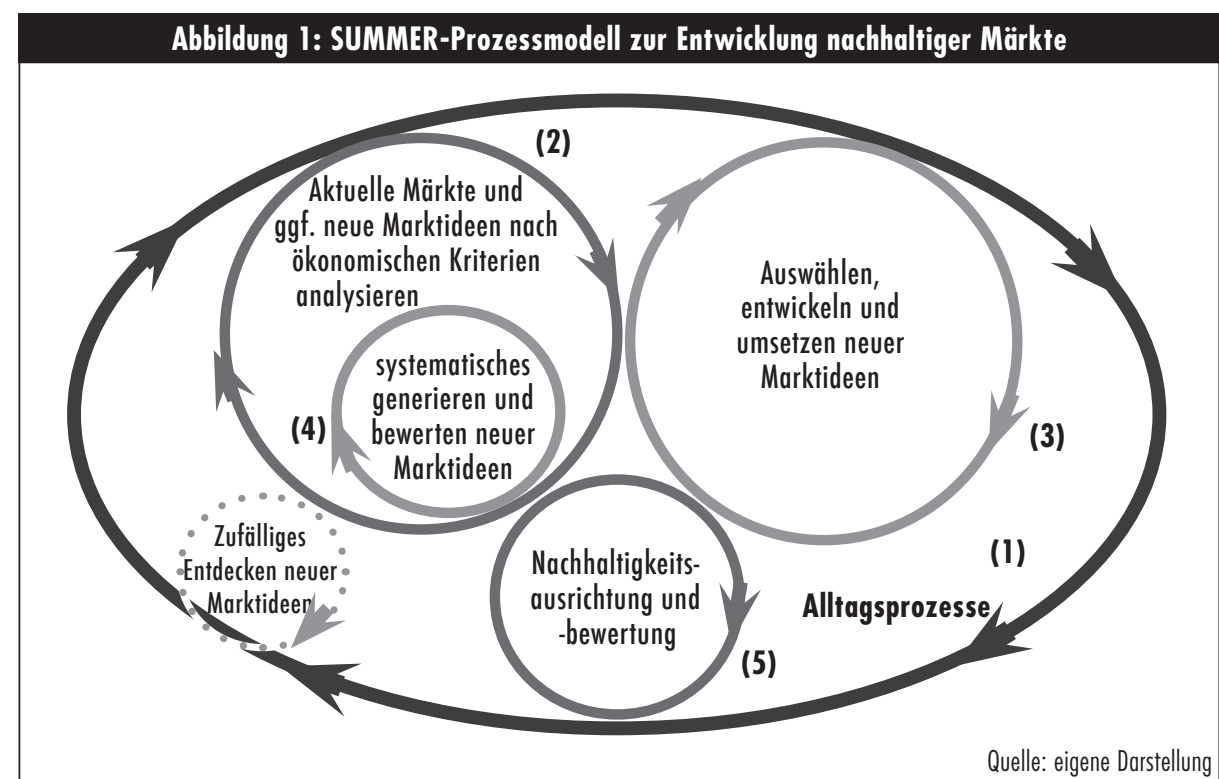




\section{- Auf der Suche nach nachhaltigen- Marktideen}

Weder der Prozess der Strategie- und Innovationsbewertung, noch der Prozess der Strategieund Innovationsumsetzung umfassen automatisch die systematische Suche nach neuen Marktideen (Grafik Punkt 4). Methoden dafür sind zum Beispiel die Szenariotechnik, Innovationsworkshops mit Leitkunden oder Zukunftswerkstätten. So kann sich ein Bewertungsprozess ausschließlich auf vorhandene Marktaktivitäten und bestimmte Modifikationen oder zufällig entstandene neue Marktideen beschränken. Dies geschieht etwa mit Hilfe der Portfoliotechnik, einer Branchenstrukturanalyse oder wie bei Velotaxi mit intensiver Kommunikation zwischen Velotaxi und den internationalen Partnerbetrieben sowie einer systematischen Sammlung und Besprechung neuer Ideen im Rahmen von Strategie- und Vertriebstreffen. Realisierungsprozesse beginnen mit einer Entscheidung zwischen bereits vorliegenden Marktideen. Im Realisierungsprozess muss dann immer wieder die Marktfähigkeit eines neuen Angebots bewertet und diese gegebenenfalls durch Veränderungen erhöht werden. Dabei wird jedoch nicht mehr systematisch nach gänzlich alternativen Marktideen gesucht. Dies würde den Umsetzungsprozess nur behindern. Die systematische Suche nach neuen Marktideen stellt daher einen eigenen Prozess dar, den wir aufgrund seines engen Bezugs zur ökonomischen Bewertung von Marktideen als mögliche Erweiterung in den Teilprozess des strategischen Managements integrieren. Beispielhaft dafür kann der SUMMERPraxispartner Mohn Media als Unternehmen auf dem Markt für Druckereierzeugnisse angeführt werden. Um permanent auf dem durch eine extrem hohe Konkurrenzsituation geprägten Markt zu bestehen, generiert und bewertet Mohn Media systematisch neue Marktideen. Zu diesen zählen im Bereich Produktinnovation insbesondere Papiere mit höherem Sekundärfaseranteil, im Bereich Prozessinnovation vor allem Druckprozesse mit weniger Papierabfällen. Zudem werden auch systematisch Neuerungen in der Papierkette entwickelt.

Werden Innovationen im Hinblick auf Nachhaltigkeitskriterien betrachtet, stehen deren Nachhaltigkeitspotenzial und deren tatsächlicher Nachhaltigkeitsbeitrag im Vordergrund. Inwieweit positive Nachhaltigkeitseffekte einzelner Innovationen beabsichtigt sind und explizit angestrebt wurden, ist bezogen auf den tatsächlichen Nachhaltigkeitsbeitrag jedoch zunächst sekundär. Für das Verständnis und die Gestaltung von Nachhaltigkeitsinnovationen spielen diese Aspekte allerdings eine bedeutende Rolle. Unabhängig von Motivationen und Intentionen zu nachhaltigem unternehmerischem Verhalten bedarf es für eine Entwicklung in Richtung Nachhaltigkeit zahlreicher aktiver Suchprozesse. Anders ausgedrückt: Neuerungen mit einem Nachhaltigkeitsbeitrag können ohne aktive Suche und nicht generiert werden; eine willentliche Entwicklung von Nachhaltigkeitsinnovationen beziehungsweise Generierung neuer nachhaltigkeitsorientierter Märkte setzt allerdings ein Bewusstsein und Bewusstwerden über die zielgerichtete Suche voraus. Ebenso wie der Prozess der systematischen Suche nach neuen Marktideen stellt auch der Prozess der Nachbaltigkeitsausrichtung und -bewertung längst keinen Automatismus im Rahmen unternehmerischer Handlungsabläufe dar (Grafik Punkt 5). Die explizite Orientierung an Nachhaltigkeitskriterien und die dementsprechende Bewertung von Handlungsalternativen kann dabei sowohl an Alltagsprozesse wie auch an explizite Bewertungs- oder Realisierungsprozesse anknüpfen. Dies hat etwa die Firma KonnexX im Rahmen der Entwicklung und Realisierung eines neuen zukunftsfähigen Möbelprogramms vorgenommen. Mit Hilfe des Innovations-Radars ${ }^{\circledR}$ wurden die Anforderungsprofile der Kunden, Hersteller und Zulieferer an das zukunftsfähige Möbelprogramm mit Nachhaltigkeitsanforderungen abgestimmt. Letztere umfassen unter anderem Auswahl von Materialien mit geringen Umwelteinwirkungen, Verringerung des Materialeinsatzes, Optimierung der Produktionstechniken sowie des Distributionssystems, Verringerung der Umwelteinwirkungen während der Nutzungsphase, Optimierung der Lebens-/ Nutzungsdauer und der Rückführungs- und Entsorgungsphase sowie die Entwicklung eines neuen Produktkonzeptes.

\section{Fazit}

Für die gezielte Entwicklung nachhaltiger $\mathrm{Zu}$ kunftsmärkte ist zweifellos unerlässlich, neben den Alltagsprozessen auch die expliziten Teilprozesse zu durchlaufen. Das Prozessmodell macht deutlich, dass diese expliziten Prozesse sowohl untereinander als auch mit den Basisprozessen auf sehr verschiedene Weise verknüpft sein können und sollten. Die so gewonnene Transparenz über die verschiedenen Teilprozesse und deren Berührungspunkte hilft auf der Forschungsseite, den
Gegenstand unternehmerischer Entwicklungsprozesse zu lokalisieren und verschiedene Teilprozesse sinnvoll abzugrenzen. Für die Unternehmenspraxis dient sie dazu, die Kommunikation und kritische Reflexion über die Schwerpunkte der eigenen Strategie- und Innovationsprozesse anzuregen und nach Veränderungsansätzen in diesen Prozessen und ihrem Zusammenwirken zu fragen.

Von besonderem Interesse ist dabei die Klärung, ob einzelne Teilprozesse überproportional ausgeprägt sind, während andere übersehen werden. So zählt in vielen reiferen, oftmals mittelständischen Unternehmen die systematische Suche nach neuen Marktideen und eine damit verbundene Nachhaltigkeitsorientierung und -bewertung zu den deutlich vernachlässigten Teilprozessen. $\mathrm{Zu}$ fragen ist, mit welchen Interaktions- und Analysemethoden eine sinnvolle Kopplung und Bezugnahme dieser Teilprozesse geleistet werden kann und welche Voraussetzungen nötig sind, damit Unternehmen zu dieser Kopplung bereit sind. In jungen Unternehmen verschmilzt dagegen oft die Realisierung immer wieder neuer, oft eher zufälliger Marktideen mit Alltagsprozessen. Hier kann das Modell klären helfen, ob eine Stärkung des Teilprozesses Marktbewertung die Chance bietet, insgesamt in eine gesunde Balance der Teilprozesse zu gelangen. Ziel ist nämlich nicht allein die kluge Umsetzung einzelner Teilprozesse, sondern immer auch deren angemessene Balance. Unternehmen können demnach vielfältig entsprechende Prozesse durchlaufen und aktive Nachhaltigkeitsbeiträge leisten, sich als Akteure im Rahmen einer nachhaltigen Entwicklung positionieren und mit ihrem Reflektions- und Innovationsverhalten eine Brücke zur Nachhaltigkeit schlagen. Und vielleicht schlagen Sie am besagten Morgen im Jahr 2022 Ihre morgendliche Zeitung auf und lesen dann ...

\section{Die Autorlnnen}

Dipl.-Kffr. Marlen Arnold und Dr. Andreas Aulinger sind Mitarbeiter im Projekt SUMMER am Lehrstuhl für ABWL, Unternehmensführung und Betriebliche Umweltpolitik an der Universität Oldenburg.

Kontakt: Universität Oldenburg, Fakultät II, Lehrstuhl für ABWL, Unternehmensführung und Betriebliche Umweltpolitik, 26111 Oldenburg. Tel. 0441-7984184,

E-Mail: marlen.arnold@uni-oldenburg.de; andreas.aulinger@uni-oldenburg.de 
(c) 20I0 Authors; licensee IÖW and oekom verlag. This is an article distributed under the terms of the Creative Commons Attribution Non-Commercial No Derivates License (http://creativecommons.org/licenses/by-nc-nd/3.o/), which permits unrestricted use, distribution, and reproduction in any medium, provided the original work is properly cited. 